\title{
On the structure of the post-Newtonian expansion in general relativity
}

\author{
Luc Blanchet * Guillaume Faye $t$ and Samaya Nissanket \\ $\mathcal{G} \mathbb{R} \varepsilon \mathbb{C O}$ - Gravitation et Cosmologie, \\ Institut d'Astrophysique de Paris, C.N.R.S., \\ $98^{\text {bis }}$ boulevard Arago, 75014 Paris, France
}

(Dated: October 28, 2018)

\begin{abstract}
In the continuation of a preceding work, we derive a new expression for the metric in the near zone of an isolated matter system in post-Newtonian approximations of general relativity. The post-Newtonian metric, a solution of the field equations in harmonic coordinates, is formally valid up to any order, and is cast in the form of a particular solution of the wave equation, plus a specific homogeneous solution which ensures the asymptotic matching to the multipolar expansion of the gravitational field in the exterior of the system. The new form is suitable for practical computations of the gravitational radiation reaction forces in harmonic coordinates. It also provides some insights on the structure of the post-Newtonian expansion in general relativity and the radiation reaction terms therein.

PACS numbers: 04.25.Nx, 04.30.--w
\end{abstract}

\footnotetext{
*Electronic address: blanchet@iap.fr

${ }^{\dagger}$ Electronic address: faye@iap.fr

${ }^{\ddagger}$ Electronic address: nissanke@iap.fr
} 


\section{INTRODUCTION}

In a previous paper [1] (hereafter referred to as paper I), the equations of motion of compact binary systems were computed at the 3.5 post-Newtonian (PN) order, corresponding to the formal $1 / c^{7}$ level in a post-Newtonian expansion with respect to the Newtonian acceleration (where $c$ is the speed of light). This confirmed and extended previous calculations in Refs. [2, 3, 4, 5]. The 3.5PN terms consist of gravitational radiation reaction terms, $1 \mathrm{PN}$ order beyond the lowest order of radiation reaction, which occurs at $2.5 \mathrm{PN}$ order in the acceleration. To deal with the divergences of Poisson or Poisson-like integrals in higher orders (a well-known problem in the standard post-Newtonian formalism), paper I used a particular solution of the Poisson equation defined by means of a finite part operation resorting to complex analytic continuation.

The aim of the present paper is to justify fully the procedure that was employed in paper I to compute high-order post-Newtonian terms (in particular the radiation reaction terms therein), and that was based on a formal expansion of the retardation of the standard retarded integral, augmented by the finite part operation. The proof will essentially consist of deriving a new expression for the post-Newtonian expansion in the near zone of a general isolated post-Newtonian source, which will constitute an interesting variant of the solution proposed in a preceding article [6] (hereafter paper II). The latter solution is obtained within a particular framework combining the multipolar-post-Minkowskian formalism with a matching to a post-Newtonian source [7, 8, 9].

More precisely, in paper II, a general expression is provided for the gravitational field generated by an isolated (slowly-moving) system in its near zone, developed up to any postNewtonian order. This expression is free of the well-known divergences of Poisson-type integrals which had plagued the post-Newtonian expansion at high-order approximations in earlier works (see e.g. Refs. 10, 11, 12, 13, 14, 15, 16, 17] for discussions). The method adopted in paper II to deal with these divergences has been to replace the Poisson integrals by a new type of solution to the hierarchy of post-Newtonian equations. It involves a "regularization" of the boundary of the Poisson integral at infinity by means of the finite part. Let us emphasize that the finite part is only used as a mathematical trick to find in a convenient way a particular solution of the differential equation we want to solve. To this particular solution, we add a homogeneous solution which is determined by asymptotic 
matching to the external field. It is then possible to iterate formally the post-Newtonian expansion ad infinitum.

The matching must be understood as a variant of the method of asymptotic-expansion matching applicable in the exterior part of the source's near zone [9, 18, 19, 20, 21]. As the exterior solution satisfies a condition of no-incoming radiation, the solution of paper II incorporates the effects of radiation reaction and, therefore, gives a correct physical description of the isolated system.

The alternative form that we derive for the end result of paper II further clarifies its relationship with the usual method of computing the successive post-Newtonian approximations, based on a naive expansion of the field equations, like for instance in Ref. [13]. Essentially, we show that the present method leads to correct results up to the 3.5PN order; this is, however, no longer the case starting from the $4 \mathrm{PN}$ order $\sim 1 / c^{8}$, because of the nonlinear contributions of gravitational wave tails [20, 22, 23]. As we shall see, the expression

of the post-Newtonian expansion derived below provides further insights on the structure of the gravitational radiation reaction terms and the contribution of tails in the near zone.

Section II recalls some necessary results from paper II, and offers complementary comments. In Section III we derive the novel expression for the post-Newtonian expansion, which we subsequently discuss. Finally, in Section IV] we check directly, by using our new form, that this solution is divergenceless, and thus satisfies the harmonic coordinate Einstein field equations. The paper ends in Section $\nabla$ with a short conclusion.

\section{REMINDERS OF EARLIER WORK AND COMMENTS}

In the following, we shall describe the gravitational field in harmonic coordinates, assumed to exist globally and to be unique, in terms of the "Gothic" metric deviation from flat spacetime, $h^{\mu \nu}$. We shall thus have

$$
\begin{aligned}
& \partial_{\nu} h^{\mu \nu}=0, \\
& h^{\mu \nu} \equiv \sqrt{-g} g^{\mu \nu}-\eta^{\mu \nu},
\end{aligned}
$$

where $g^{\mu \nu}$ and $g$ are the inverse matrix and the determinant of the usual covariant metric $g_{\alpha \beta}$ respectively, and where $\eta^{\mu \nu}=\operatorname{diag}(-1,1,1,1)$ denotes the Minkowski flat metric in Cartesian coordinates. With this choice and notation, the "relaxed" Einstein field equations 
simply read

$$
\square h^{\mu \nu}=\frac{16 \pi G}{c^{4}} \tau^{\mu \nu}
$$

with $\square \equiv \eta^{\alpha \beta} \partial_{\alpha \beta}$ being the flat d'Alembertian and $G$ the Newtonian constant. The pseudo stress-energy tensor $\tau^{\mu \nu}$, accounting for the matter as well as the gravitational fields in harmonic coordinates, is given by

$$
\tau^{\mu \nu}=(-g) T^{\mu \nu}+\frac{c^{4}}{16 \pi G} \Lambda^{\mu \nu}\left[h^{\alpha \beta}\right]
$$

where $T^{\mu \nu}$ is the stress-energy tensor of the extended localized (bounded) matter source with spatially compact support, and $\Lambda^{\mu \nu}\left[h^{\alpha \beta}\right]$ is the gravitational stress-energy distribution, which depends non-linearly on $h^{\alpha \beta}$ and its space-time derivatives. The pseudo-tensor is conserved in the usual sense:

$$
\partial_{\nu} \tau^{\mu \nu}=0
$$

Now, we are interested in the general expression of the post-Newtonian expansion valid in the source's near zone, i.e. for the coordinate distance $r$ significantly smaller than the gravitational wave length of the emitted radiation. The field equations (2.1)-(2.2), supplemented by a condition of no-incoming radiation, are solved iteratively as a formal expansion when $c \rightarrow+\infty$. The post-Newtonian series of the field variable $h^{\mu \nu}$, denoted by means of an overbar, $\bar{h}^{\mu \nu}=\mathrm{PN}\left[h^{\mu \nu}\right]$, takes the following "polylogarithmic" form [7]:

$$
\bar{h}^{\mu \nu}(\mathbf{x}, t, c)=\sum \frac{(\ln c)^{q}}{c^{p}} h_{p, q}^{\mu \nu}(\mathbf{x}, t)
$$

with post-Newtonian coefficients $h_{p, q}^{\mu \nu}(p, q \in \mathbb{N})$ depending on the field point $\mathbf{x}$ and coordinate time $t$. The pseudo-tensor, $\tau^{\mu \nu}$, admits a post-Newtonian expansion $\bar{\tau}^{\mu \nu}$ of the same type, say with coefficients $\tau_{p, q}^{\mu \nu}$. The post-Newtonian expansions $\bar{h}^{\mu \nu}$ and $\bar{\tau}^{\mu \nu}$ are connected via the field equations by

$$
\square \bar{h}^{\mu \nu}=\frac{16 \pi G}{c^{4}} \bar{\tau}^{\mu \nu}
$$

completed with the harmonic gauge condition

$$
\partial_{\nu} \bar{h}^{\mu \nu}=0
$$

In the present paper, we shall not consider expressions of the post-Newtonian coefficients at a given order, but rather work with whole post-Newtonian expansions $\bar{h}^{\mu \nu}$ and $\bar{\tau}^{\mu \nu}$ of the type (2.5). Such expansion series will be manipulated by means of the rules of formal 
series. In particular, we shall not investigate the eventual convergence properties, or, more generally, the mathematical nature of these series. The developments presented in paper II and the present one will thus constitute some formal generalization, to any post-Newtonian order, of the expressions of the near zone metric and the radiation reaction force. These expressions have been explicitly validated by detailed computations at $2 \mathrm{PN}$ order for the matching process as well as the multipole moments [8], and at $1.5 \mathrm{PN}$ relative order for the radiation reaction contributions 22,23$]$.

The strategy adopted in paper II was (i) to solve the wave equation (2.6) in the form of a certain functional of the pseudo tensor, $\bar{h}^{\mu \nu}\left[\tau^{\alpha \beta}\right]$, and (ii) to verify that the gauge condition (2.7) is satisfied as a consequence of the conservation of $\tau^{\mu \nu}$, Eq. (2.4). Importantly, the post-Newtonian expansion of the gravitational field, $\bar{h}^{\mu \nu}$, was found to be a functional not only of the post-Newtonian expansion of the pseudo-tensor, $\bar{\tau}^{\mu \nu}$, but also of its multipole expansion, denoted below by $\mathcal{M}\left(\tau^{\mu \nu}\right)$, which is valid in the source's exterior, and in particular in the asymptotic regions at infinity. This can be understood by the fact that the postNewtonian solution (comprising the radiation reaction terms) depends on the boundary conditions imposed at infinity, especially the no-incoming radiation condition at past null infinity, which must describe a matter system isolated from the rest of the universe.

In the formalism of paper II, the no-incoming radiation condition is trivially satisfied, because it is assumed that the matter source is stationary before a finite date in the remote past, say, for $t \leq-\mathcal{T}$, with $-\mathcal{T}$ denoting a fixed instant in the past. More precisely, we suppose that both $h^{\mu \nu}$ and $\tau^{\mu \nu}$ are constant (with respect to time) outside the causal future development of the source when it starts emitting radiation, i.e. for instance

$$
\tau^{\mu \nu}(\mathbf{x}, t)=\tau_{\mathrm{s}}^{\mu \nu}(\mathbf{x}) \text { when } t-\frac{|\mathbf{x}|}{c} \leq-\mathcal{T}
$$

where the function $\tau_{\mathrm{s}}^{\mu \nu}(\mathbf{x})$ does not depend on $t$, and refers to the "stationary" value taken by the pseudo stress-energy tensor at early times. In the present paper, we shall continue to assume that Eq. (2.8) and the same equation for $h^{\mu \nu}$ hold. It will be convenient to decompose the pseudo tensor into the sum of its stationary value, defined by Eq. (2.8), and a "non-stationary" part, denoted by $\tau_{\mathrm{ns}}^{\mu \nu}(\mathbf{x}, t)$, that vanishes when $t \leq-\mathcal{T}$. Hence, we pose, at any time $t$,

$$
\tau^{\mu \nu}(\mathbf{x}, t)=\tau_{\mathrm{s}}^{\mu \nu}(\mathbf{x})+\tau_{\mathrm{ns}}^{\mu \nu}(\mathbf{x}, t)
$$

Such splitting is always possible thanks to the hypothesis of past stationarity. In this 
formalism, the derivation of any physical result is to be done under the assumption (2.8), but at the end of the calculation, once the result is in hand, $\mathcal{T}$ can be sent to $+\infty$.

The main outcome of paper II is that the post-Newtonian solution of the wave equation (2.6), and satisfying (2.7), together with correct boundary conditions at infinity, may be written as the sum of a particular (inhomogeneous) solution plus a specific homogeneous solution; both of which are now described.

Let us first consider the particular solution. It is given by the so-called operator of instantaneous potentials, denoted by $\mathcal{I}^{-1}$, which is defined by a straightforward iteration of the inverse Poisson operator $\Delta^{-1}$. The instantaneous operator $\mathcal{I}^{-1}$ is endowed with a regularization, dealing with the boundary of the integrals at infinity, i.e. for $\left|\mathbf{x}^{\prime}\right| \rightarrow$ $+\infty$. This regularization is defined from a particular finite part procedure, indicated by an operator $\mathrm{FP}_{B=0}$, with analytic continuation in the parameter $B \in \mathbb{C}$ down to $B=0$. Essentially, it consists in multiplying the integrand of the Poisson integral by a factor $\left|\mathbf{x}^{\prime}\right|^{B}$, and taking the finite part of the Laurent expansion of the (analytic continuation of the) integral when $B \rightarrow 0$. The analytic continuation is always possible at a given post-Newtonian order for the particular class of functions considered in paper II as well as in the present paper ( $c f$. Appendix B in paper II). Thanks to this particular regularization, ${ }^{1}$ we can select a solution of the iterated Poisson equations, that is different from the standard Poisson-type integral and avoids the appearance of divergences at the boundary at infinity (see paper II for the details). The crucial point is that the latter construction is a particular solution of these equations. We thus have

$$
\widetilde{\mathcal{I}^{-1}}\left[\bar{\tau}^{\mu \nu}\right]=\sum_{k=0}^{+\infty}\left(\frac{\partial}{c \partial t}\right)^{2 k} \widetilde{\Delta^{-k-1}}\left[\bar{\tau}^{\mu \nu}\right],
$$

where the $k$-th iterated Poisson integral reads

$$
\widetilde{\Delta^{-k-1}}\left[\bar{\tau}^{\mu \nu}\right](\mathbf{x}, t)=\widetilde{\int} \frac{d^{3} \mathbf{x}^{\prime}}{-4 \pi} \frac{\left|\mathbf{x}-\mathbf{x}^{\prime}\right|^{2 k-1}}{(2 k) !} \bar{\tau}^{\mu \nu}\left(\mathbf{x}^{\prime}, t\right) .
$$

The overtilde on a quantity means here (following the notation of paper II) regularization by use of the finite part operation $\mathrm{FP}_{B=0}$ :

$$
\widehat{\Delta^{-k-1}}[f(\mathbf{x})] \equiv \underset{B=0}{\mathrm{FP}} \Delta^{-k-1}\left[\left|\mathbf{x} / r_{0}\right|^{B} f(\mathbf{x})\right]
$$

\footnotetext{
${ }^{1}$ Note that although it is sometimes convenient to call it as such, the scheme is strictly speaking not a regularization since there is no assumption that some infinite quantity should be formally discarded.
} 


$$
\widetilde{\int} d^{3} \mathbf{x}^{\prime} g\left(\mathbf{x}^{\prime}\right) \equiv \underset{B=0}{\mathrm{FP}} \int d^{3} \mathbf{x}^{\prime}\left|\mathbf{x}^{\prime} / r_{0}\right|^{B} g\left(\mathbf{x}^{\prime}\right) .
$$

Following earlier articles (Refs. [8, 9] and paper II), we introduce an independent length scale $r_{0}$ inside the regularization factor in order to adimensionalize it. Anyway, we know that the constant $r_{0}$ disappears from the physical metric as soon as the metric is functionally related to the local matter distribution. As shown in paper II, Eq. (2.10) indeed constitutes a solution of the d'Alembertian equation (2.6):

$$
\square\left\{\widetilde{\mathcal{I}^{-1}}\left[\bar{\tau}^{\mu \nu}\right]\right\}=\bar{\tau}^{\mu \nu}
$$

We next add a homogeneous solution in such a way that the post-Newtonian metric matches the external one, which is globally defined outside the source in the form of a multipolar expansion. Clearly, such a homogeneous solution must be regular inside the matter source, and in particular for $r \rightarrow 0$, assuming that $r=0$ corresponds to the spatial origin with respect to which the multipolar expansion is defined. Therefore, the homogeneous solution must be made up of a sum of retarded (multipolar) waves minus the corresponding advanced waves; it is well known that such anti-symmetric waves are regular in a neighborhood of $r=0$, and are associated with radiation reaction effects. ${ }^{2}$ In the Fourier representation, their contribution takes the form of a superposition of plane monochromatic waves (see below). However, following paper II, we prefer a parametrization based on certain multipole-moment functions in the physical domain.

In paper II, we showed that, when the particular solution is taken to be that of the instantaneous operator, defined above by Eq. (2.10), the multipole-moment functions parametrizing the homogeneous solution, say $\mathcal{A}_{L}^{\mu \nu}(t)$, split in fact into a sum of two terms, ${ }^{3}$

$$
\mathcal{A}_{L}^{\mu \nu}(t)=\mathcal{F}_{L}^{\mu \nu}(t)-4 \pi \mathcal{R}_{L}^{\mu \nu}(t)
$$

The first of these functions, $\mathcal{F}_{L}^{\mu \nu}(t)$, turns out to be equal to the actual source multipole

\footnotetext{
${ }^{2}$ Here, the qualifier "anti-symmetric" refers to the formal structure of the waves, i.e. retarded minus advanced, but not to their real behavior under a time reversal, which can be very complicated in high post-Newtonian approximations.

${ }^{3}$ Here, $L=i_{1} \cdots i_{\ell}$ stands for a multi-index composed of $\ell$ spatial indices $(\ell$ being often referred to as the multipolar order). For the second term in Eq. (2.14), we employ a slightly different notation than in paper II, where it is given by $\mathcal{R}_{L}^{\mu \nu}$ (paper II) $=-4 \pi \mathcal{R}_{L}^{\mu \nu}$.
} 
moment of the isolated system, as seen from the exterior region [9]. It is given by

$$
\mathcal{F}_{L}^{\mu \nu}(t)=\widetilde{\int} d^{3} \mathbf{x}^{\prime} \hat{x}_{L}^{\prime} \int_{-1}^{+1} d z \delta_{\ell}(z) \bar{\tau}^{\mu \nu}\left(\mathbf{x}^{\prime}, t-z\left|\mathbf{x}^{\prime}\right| / c\right)
$$

with $\hat{x}_{L}^{\prime}=\operatorname{STF}\left(x_{i_{1}}^{\prime} \cdots x_{i_{\ell}}^{\prime}\right)$ denoting the symmetric-trace-free (STF) part of the product of $\ell$ spatial vectors; the function $\delta_{\ell}(z)$, whose integral is normalized to unity, is defined by

$$
\begin{gathered}
\delta_{\ell}(z)=\frac{(2 \ell+1) ! !}{2^{\ell+1} \ell !}\left(1-z^{2}\right)^{\ell}, \\
\int_{-1}^{+1} d z \delta_{\ell}(z)=1 .
\end{gathered}
$$

We emphasize that expression (2.15), together with (2.16), is physically valid only for postNewtonian sources. As such, it must be considered in a perturbative sense and given as some formal post-Newtonian expansion. The explicit post-Newtonian series reads (see paper II)

$$
\mathcal{F}_{L}^{\mu \nu}(t)=\sum_{j=0}^{+\infty}\left(\frac{d}{c d t}\right)^{2 j} \widetilde{\int} d^{3} \mathbf{x}^{\prime} \widetilde{\Delta^{-j}}\left(\hat{x}_{L}^{\prime}\right) \bar{\tau}^{\mu \nu}\left(\mathbf{x}^{\prime}, t\right),
$$

where we have introduced the convenient notation

$$
\widetilde{\Delta^{-j}}\left(\hat{x}_{L}^{\prime}\right)=\frac{(2 \ell+1) ! !}{2^{j} j !(2 \ell+2 j+1) ! !}\left|\mathbf{x}^{\prime}\right|^{2 j} \hat{x}_{L}^{\prime},
$$

which is justified by the fact that $\Delta^{j}\left[\widetilde{\Delta^{-j}}\left(\hat{x}_{L}^{\prime}\right)\right]=\hat{x}_{L}^{\prime}$.

As can be seen from Eqs. (2.15) or (2.17), the function $\mathcal{F}_{L}^{\mu \nu}$ is defined as a functional of the post-Newtonian expansion of the pseudo-tensor $\bar{\tau}^{\mu \nu}$, valid in the source's near zone. The second piece of the multipolar function (2.14), namely $\mathcal{R}_{L}^{\mu \nu}$, directly issues from the matching process to the external field, and arises purely from non-linearities. Thus, it depends on the expression of the pseudo-tensor in the exterior of the source (including the region located at infinity), where it is given in the form of a multipolar series $\mathcal{M}\left(\tau^{\mu \nu}\right)$, with $\mathcal{M}$ representing the multipolar expansion. Actually, in the present formalism, $\mathcal{M}\left(h^{\mu \nu}\right)$, and $\mathcal{M}\left(\tau^{\mu \nu}\right)$ as well, are identified with the so-called multipolar-post-Minkowskian expansion of the external field, in the sense of Ref. 7]. ${ }^{4}$ The function $\mathcal{R}_{L}^{\mu \nu}$ may be written in a compact way as (see [22]

\footnotetext{
${ }^{4}$ The post-Minkowskian expansion (or non-linearity expansion when $G \rightarrow 0$ ) plays a crucial role in the construction of the multipolar-post-Minkowskian solution in Ref. 7], but shall not be considered for the present purpose. Indeed, we shall assume here that we are always entitled to sum up formally the post-Minkowskian series and to treat the formal limit as if it were "exact".
} 
and paper II)

$$
\mathcal{R}_{L}^{\mu \nu}(t)=\widetilde{\int} \frac{d^{3} \mathbf{x}^{\prime}}{-4 \pi} \hat{x}_{L}^{\prime} \int_{1}^{+\infty} d z \gamma_{\ell}(z) \mathcal{M}\left(\tau_{\mathrm{ns}}^{\mu \nu}\right)\left(\mathbf{x}^{\prime}, t-z\left|\mathbf{x}^{\prime}\right| / c\right)
$$

where $\gamma_{\ell}(z) \equiv-2 \delta_{\ell}(z)$ is such that

$$
\int_{1}^{+\infty} d z \gamma_{\ell}(z)=1
$$

Remark that the above normalization of the integral of $\gamma_{\ell}(z)$ is a consequence of that of $\delta_{\ell}(z)\left[\right.$ Eq. (2.16b)], together with the fact that $\int_{-\infty}^{+\infty} d z \gamma_{\ell}(z)=0$ by analytic continuation in the variable $\ell \in \mathbb{C}$.

Note that, as indicated in Eq. (2.19), the function $\mathcal{R}_{L}^{\mu \nu}$ involves the non-stationary part of the pseudo-tensor, $\tau_{\mathrm{ns}}^{\mu \nu}$, given by Eqs. (2.8)-(2.9); hence the integral over $z$ is well-defined, as it ranges in fact on a finite domain $\left[1, z_{\max }\right]$, where $z_{\max }+1=c(t+\mathcal{T}) /\left|\mathbf{x}^{\prime}\right|$. This means that $\mathcal{R}_{L}^{\mu \nu}$ depends on the initial instant of emission, $-\mathcal{T}$, but since it appears only through an anti-symmetric type wave, in Eq. (3.7) below, the dependence on $-\mathcal{T}$ finally cancels out.

One can show that an alternative form for $\mathcal{R}_{L}^{\mu \nu}$ is

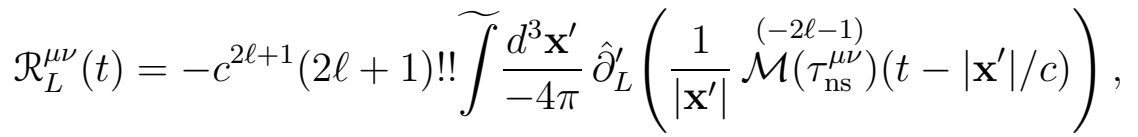

where $\hat{\partial}_{L}^{\prime}$ is the STF multi-derivative operator associated with the integration point $\mathbf{x}^{\prime}$, and the superscript $(-2 \ell-1)$ over $\mathcal{M}\left(\tau_{\mathrm{ns}}^{\mu \nu}\right)$ denotes the $(2 \ell+1)$-th time anti-derivative that vanishes in the remote past, say, when $t \leq-\mathcal{T}$. The STF spatial multi-derivative admits the following explicit expression:

$$
\hat{\partial}_{L}\left(\frac{f(t-r / c)}{r}\right)=(-1)^{\ell} \hat{n}_{L} \sum_{i=0}^{\ell} \frac{(\ell+i) !}{2^{i} i !(\ell-i) !} \frac{f^{(\ell-i)}(t-r / c)}{c^{\ell-i} r^{i+1}},
$$

where $r=|\mathbf{x}|, \hat{n}_{L}=\hat{x}_{L} / r^{\ell}, \hat{\partial}_{L}=\operatorname{STF}\left(\partial_{i_{1}} \cdots \partial_{i_{\ell}}\right)$; the superscript $(\ell-i)$ means the $(\ell-i)$-th time derivative.

With $\mathcal{A}_{L}^{\mu \nu}$ being the sum of these functions as given by Eq. (2.14), the post-Newtonian expansion in the form obtained using the method of matched asymptotic expansions in paper II reads:

$$
\bar{h}^{\mu \nu}=\frac{16 \pi G}{c^{4}} \widetilde{\mathcal{I}^{-1}}\left[\bar{\tau}^{\mu \nu}\right]-\frac{4 G}{c^{4}} \sum_{\ell=0}^{+\infty} \frac{(-1)^{\ell}}{\ell !} \hat{\partial}_{L}\left\{\frac{\mathcal{A}_{L}^{\mu \nu}(t-r / c)-\mathcal{A}_{L}^{\mu \nu}(t+r / c)}{2 r}\right\} .
$$

The explicit expression of the multipolar waves can be written from Eq. (2.22) (with $c \rightarrow-c$ in the case of the advanced wave), but we shall give some alternative formulas in Eqs. (3.1) and (3.13) below. 


\section{NEW EXPRESSION OF THE POST-NEWTONIAN EXPANSION}

The idea for determining the new form of the post-Newtonian expansion is based on the remark that, in Eq. (2.23), the part of the multipolar anti-symmetric wave that is associated with the function $\mathcal{F}_{L}^{\mu \nu}$ corresponds in fact to the "odd" parity part (i.e., the part composed of odd powers of $1 / c$ ) of the retardation expansion in the standard retarded integral. Since the operator of the instantaneous potentials (2.10)-(2.11) precisely equates the corresponding "even" part, we can reconstitute the complete retarded integral of the post-Newtonian source term in a nice way.

To begin with, we notice that the post-Newtonian expansion of the multipolar antisymmetric wave reads

$$
\hat{\partial}_{L}\left\{\frac{\mathcal{F}_{L}^{\mu \nu}(t-r / c)-\mathcal{F}_{L}^{\mu \nu}(t+r / c)}{2 r}\right\}=-\frac{1}{(2 \ell+1) ! !} \sum_{p=0}^{+\infty} \widetilde{\Delta^{-p}}\left(\hat{x}_{L}\right)\left(\frac{d}{c d t}\right)^{2 p+2 \ell+1} \mathcal{F}_{L}^{\mu \nu}(t),
$$

with notation (2.18) . Next, we recall that the function $\mathcal{F}_{L}^{\mu \nu}(t)$ itself is given by the expression (2.15) or alternatively by the post-Newtonian series (2.17). After inserting Eq. (2.17) into (3.1), we are led to a rather complicated double expansion. Furthermore, we have to sum up the latter result over all multipolarities $\ell=0, \cdots,+\infty$. At this point, let us change in an appropriate way the summation indices (posing in particular $k=j+p+\ell$ ). We are then able to recognize a double summation which turns out to be nothing but the expression of the quantity $\left|\mathbf{x}-\mathbf{x}^{\prime}\right|^{2 k}$, when developed in terms of STF harmonics. It can be written in a particularly economic form according to the following formula, in which we resort again to notation (2.18):

$$
\frac{\left|\mathbf{x}-\mathbf{x}^{\prime}\right|^{2 k}}{(2 k+1) !}=\sum_{\ell=0}^{k} \frac{(-1)^{\ell}}{\ell !(2 \ell+1) ! !} \sum_{p=0}^{k-\ell} \widetilde{\Delta^{-p}}\left(\hat{x}_{L}\right) \widetilde{\Delta^{-k+\ell}+p}\left(\hat{x}_{L}^{\prime}\right)
$$

This formula can be viewed as a finite expansion in terms of Legendre polynomials, since the product $\widetilde{\Delta^{-p}}\left(\hat{x}_{L}\right) \widetilde{\Delta^{-q}}\left(\hat{x}_{L}^{\prime}\right)$ (where $p, q \in \mathbb{N}$ ) is proportional to $\hat{x}_{L} \hat{x}_{L}^{\prime}$, and the latter is known to be in turn proportional to the $\ell$-th Legendre polynomial of the scalar products of the unit vectors $\mathbf{n}=\mathbf{x} / r$ and $\mathbf{n}^{\prime}=\mathbf{x}^{\prime} / r^{\prime}$, namely $P_{\ell}\left(\mathbf{n} \cdot \mathbf{n}^{\prime}\right)$. Thanks to Eq. (3.2), we arrive at the interesting relation

$\sum_{\ell=0}^{+\infty} \frac{(-1)^{\ell}}{\ell !} \hat{\partial}_{L}\left\{\frac{\mathcal{F}_{L}^{\mu \nu}(t-r / c)-\mathcal{F}_{L}^{\mu \nu}(t+r / c)}{2 r}\right\}=-\sum_{k=0}^{+\infty}\left(\frac{\partial}{c \partial t}\right)^{2 k+1} \widetilde{\int} d^{3} \mathbf{x}^{\prime} \frac{\left|\mathbf{x}-\mathbf{x}^{\prime}\right|^{2 k}}{(2 k+1) !} \bar{\tau}^{\mu \nu}\left(\mathbf{x}^{\prime}, t\right)$, 
which shows, as announced before, that the anti-symmetric wave associated with $\mathcal{F}_{L}^{\mu \nu}(t)$ is equal to the odd part [i.e. corresponding to $n=2 k+1, k \in \mathbb{N}$, in Eq. (3.4) below] of the expansion of the retarded integral as regularized by means of the finite part FP.

In order to gather terms (2.10) and (3.3), which are both present in Eq. (2.23), we need to consider the new object that is made of the formal expansion of the standard retarded (R) integral $\square_{\mathrm{R}}^{-1}$, but where each of the terms is regularized by means of the FP procedure (indicated by an overtilde) to deal with the divergences appearing on the boundary at infinity:

$$
\widetilde{\square_{\mathrm{R}}^{-1}}\left[\bar{\tau}^{\mu \nu}\right](\mathbf{x}, t) \equiv \sum_{n=0}^{+\infty} \frac{(-1)^{n}}{n !}\left(\frac{\partial}{c \partial t}\right)^{n} \widetilde{\int} \frac{d^{3} \mathbf{x}^{\prime}}{-4 \pi}\left|\mathbf{x}-\mathbf{x}^{\prime}\right|^{n-1} \bar{\tau}^{\mu \nu}\left(\mathbf{x}^{\prime}, t\right) .
$$

This object should be carefully distinguished from, say, $\square_{\mathrm{R}}^{-1}\left[\bar{\tau}^{\mu \nu}\right]$, involving the retarded integral $\square_{\mathrm{R}}^{-1}$ without any kind of regularization and with the retardations being kept unexpanded inside. Eq. (3.4) is also a priori different from the more "global" solution $\square_{\mathrm{R}}^{-1}\left[\tau^{\mu \nu}\right]=c^{4} h^{\mu \nu} /(16 \pi G)$, in which the pseudo-tensor has not been expanded in postNewtonian fashion. As we shall see below, Eq. (3.4) differs also from the post-Newtonian solution itself, i.e. $\widetilde{\square_{\mathrm{R}}^{-1}}\left[\bar{\tau}^{\mu \nu}\right] \neq c^{4} \bar{h}^{\mu \nu} /(16 \pi G)$.

Let us emphasize that Eq. (3.4) constitutes merely the definition of a formal Taylor-type post-Newtonian expansion, each term of which is built from the (also formal) post-Newtonian expansion of the pseudo-tensor. Such a definition is of interest as it corresponds to what one would intuitively think is the "natural" way of performing the post-Newtonian iteration, i.e. by Taylor expanding the retardations. Moreover, each of the terms of the series (3.4) are mathematically well defined thanks to the FP process, and can therefore be implemented in practical computations like in the one reported in Ref. 1]. The important point, and the only one which matters for our purpose, is that the definition (3.4) solves, in a post-Newtonian sense, the required wave equation, namely

$$
\square\left\{\widetilde{\square_{\mathrm{R}}^{-1}}\left[\bar{\tau}^{\mu \nu}\right]\right\}=\bar{\tau}^{\mu \nu},
$$

and constitutes therefore a legitimate particular solution for determining the general form of the post-Newtonian expansion $\bar{h}^{\mu \nu}$ as the sum of this quantity and some homogeneous solution which is necessarily of the anti-symmetric type.

Combining Eq. (3.3) with our definition of the operator of the instantaneous potentials (2.10) - (2.11), which corresponds to the even part of the retardation expansion $[i . e . n=2 k$, 
$k \in \mathbb{N}$, in Eq. (3.4)], we find

$$
\widetilde{\square_{\mathrm{R}}^{-1}}\left[\bar{\tau}^{\mu \nu}\right]=\widetilde{\mathcal{I}^{-1}}\left[\bar{\tau}^{\mu \nu}\right]-\frac{1}{4 \pi} \sum_{\ell=0}^{+\infty} \frac{(-1)^{\ell}}{\ell !} \hat{\partial}_{L}\left\{\frac{\mathcal{F}_{L}^{\mu \nu}(t-r / c)-\mathcal{F}_{L}^{\mu \nu}(t+r / c)}{2 r}\right\} .
$$

This formula is the basis of our writing of the new form of the post-Newtonian expansion. Indeed, by comparing Eqs. (2.23) and (3.6), we obtain the following expression:

$$
\bar{h}^{\mu \nu}=\frac{16 \pi G}{c^{4}}\left[\widetilde{\square_{\mathrm{R}}^{-1}}\left[\bar{\tau}^{\mu \nu}\right]+\sum_{\ell=0}^{+\infty} \frac{(-1)^{\ell}}{\ell !} \hat{\partial}_{L}\left\{\frac{\mathcal{R}_{L}^{\mu \nu}(t-r / c)-\mathcal{R}_{L}^{\mu \nu}(t+r / c)}{2 r}\right\}\right],
$$

which relates the post-Newtonian expansion of $h^{\mu \nu}=\left(16 \pi G / c^{4}\right) \square_{\mathrm{R}}^{-1}\left[\tau^{\mu \nu}\right]$ to the developed operator $\widetilde{\square_{\mathrm{R}}^{-1}}\left[\bar{\tau}^{\mu \nu}\right]$ acting on the post-Newtonian expansion of the source term, $\bar{\tau}^{\mu \nu}$. Eq. (3.7), together with the conservation law for the pseudo-tensor [Eq. (2.4)], constitute our final solution for the present problem.

Let us insist that the formula (3.7) should be understood, for practical calculations, in an iterative post-Newtonian way. At the lowest order, the source has compact support and there is no need of the finite part procedure. Higher-order equations are obtained by inserting previous iterations into the source term $\bar{\tau}^{\mu \nu}\left[\right.$ or $\left.\mathcal{M}\left(\tau^{\mu \nu}\right)\right]$ truncated at this order; the source will be of non-compact support because of the gravitational field contribution. One then multiplies it by the regularization factor $\left|\mathbf{x} / r_{0}\right|^{B}$, integrates and performs the Laurent expansion when $B \rightarrow 0$ to get the finite parts needed in the two terms of Eq. (3.7). Some examples of such finite parts of integrals can be found in paper I [see e.g. Eqs. (4.15-16) there].

Equation (3.7) is more interesting and probably more "fruitful" in applications than the old form (2.23). Indeed, recall that the anti-symmetric waves in Eq. (2.23), parametrized by $\mathcal{A}_{L}^{\mu \nu}=\mathcal{F}_{L}^{\mu \nu}-4 \pi \mathcal{R}_{L}^{\mu \nu}$, are linked with radiation reaction effects. It was noticed in paper II that the part associated with $\mathcal{F}_{L}^{\mu \nu}$ starts at the usual dominant order for the radiation reaction force, i.e. the $2.5 \mathrm{PN}$ order (where it yields the standard expression for the "Newtonian", quadrupolar radiation reaction). It includes also the next term of order 3.5PN corresponding to the mass octupole and current quadrupole radiation reaction, and probably also the subsequent contributions of higher multipole moments that parametrize the radiation reaction at the linearized level. ${ }^{5}$ On the other hand, the part linked with the functions $\mathcal{R}_{L}^{\mu \nu}$ has been

\footnotetext{
${ }^{5}$ By multipole moments here, we mean the "source" moments defined in Ref. [9], which couple together non-linearly in the expressions of the radiation reaction force and the "radiative" moments seen at infinity.
} 
shown in Ref. 22] to be due to the non-linear contribution of the gravitational wave tails in the radiation reaction force. It was determined beforehand in Ref. [20] and shown there to appear at the $4 \mathrm{PN}$ order, i.e. $1.5 \mathrm{PN}$ order relative to the dominant radiation reaction effect.

Consequently, when computing the radiation reaction force limited at the $3.5 \mathrm{PN}$ order, the part associated with $\mathcal{R}_{L}^{\mu \nu}$ can be neglected, and we can keep only the part given by the first term of Eq. (3.7); this corresponds indeed to the intuitive way of performing the postNewtonian iteration (bypassing the tail effects), which was advocated in old investigations of the post-Newtonian expansion for isolated fluids, like those of Ref. [13]. Thus, Eq. (33.7) rigorously proves that the $3.5 \mathrm{PN}$ radiation reaction must be computed by considering the odd-parity part of the retardations in the usual retarded integral, provided that the source terms be multiplied by the regularization factor $\left|\mathbf{x}^{\prime}\right|^{B}$ and the integral computed following the FP prescription when $B \rightarrow 0$. This is exactly what has been done in the paper [1] (see notably Section IV there) devoted to the computation of the $3.5 \mathrm{PN}$ radiation reaction in the case of compact binary systems. However, for higher-order computations, at the 4PN level and beyond, it is definitely necessary to take into account the contribution of the functions $\mathcal{R}_{L}^{\mu \nu}$ in Eq. (3.7) which is induced by wave tails.

For some applications, it may be useful to rephrase our result with the help of some more systematic notation. Notably, we may use an appropriate operator, denoted $\mathcal{D}^{-1}$ henceforth, which maps the source term of the Einstein field equations, namely the pseudo tensor $\tau^{\mu \nu}$, onto the post-Newtonian solution given by Eq. (3.7). Let us first recall that $h^{\mu \nu}=$ $\left(16 \pi G / c^{4}\right) \square_{\mathrm{R}}^{-1}\left[\tau^{\mu \nu}\right]$, which allows us to suppress the overall factor $16 \pi G / c^{4}$ in Eq. (3.7) and reexpress the latter equation in the form of

$$
\overline{\square_{\mathrm{R}}^{-1}\left[\tau^{\mu \nu}\right]}=\widetilde{\square_{\mathrm{R}}^{-1}}\left[\bar{\tau}^{\mu \nu}\right]+\mathcal{H}^{\mu \nu}
$$

where the specific homogeneous, anti-symmetric wave type solution is defined by

$$
\mathcal{H}^{\mu \nu} \equiv \sum_{\ell=0}^{+\infty} \frac{(-1)^{\ell}}{\ell !} \hat{\partial}_{L}\left\{\frac{\mathcal{R}_{L}^{\mu \nu}(t-r / c)-\mathcal{R}_{L}^{\mu \nu}(t+r / c)}{2 r}\right\}
$$

Eq. (3.8) suggests introducing some convenient functional operators acting on the components of $\tau^{\mu \nu}$. For the first term of Eq. (3.8), we introduce the operator $\overline{\square_{\mathrm{R}}^{-1}}$, whose action on $\tau^{\mu \nu}$ is defined by

$$
\overline{\square_{\mathrm{R}}^{-1}}\left[\tau^{\mu \nu}\right] \equiv \widetilde{\square_{\mathrm{R}}^{-1}}\left[\bar{\tau}^{\mu \nu}\right]
$$


With this notation, we include into the definition of $\overline{\square_{\mathrm{R}}^{-1}}$ both the FP process, indicated by the overtilde [see Eq. (2.12)], and the operation of taking the post-Newtonian expansion, $\bar{\tau}^{\mu \nu}=\operatorname{PN}\left[\tau^{\mu \nu}\right]$. For the second term of Eq. (3.8), we pose $\mathcal{H}\left[\tau^{\mu \nu}\right] \equiv \mathcal{H}^{\mu \nu}$, and hence $\mathcal{H}$ represents the particular functional that is obtained when the functions $\mathcal{R}_{L}^{\mu \nu}$ in Eq. (3.9) are replaced by their explicit expressions in terms of $\tau^{\mu \nu}$ as given by Eqs. (2.19) or (2.21). Note that $\mathcal{R}_{L}^{\mu \nu}$ and $\mathcal{H}^{\mu \nu}$ depend in fact on the multipole expansion $\mathcal{M}\left(\tau^{\mu \nu}\right)$ of the pseudo tensor, rather than on $\tau^{\mu \nu}$ itself. As a result, the operation of taking the multipole expansion $(\mathcal{M})$ is included into the above definition of the functional $\mathcal{H}\left[\tau^{\mu \nu}\right]$. Naturally, the latter functional is also a function of the field point $\mathbf{x}$ and of (coordinate) time $t$. Thus, our notation means more precisely $\mathcal{H}\left[\tau^{\mu \nu}\right](\mathbf{x}, t) \equiv \mathcal{H}^{\mu \nu}(\mathbf{x}, t)$. In a similar way, it is convenient to make use of what we call $\mathcal{R}_{L}$, namely a functional of $\tau^{\mu \nu}$ and a function of time $t$ defined by $\mathcal{R}_{L}\left[\tau^{\mu \nu}\right](t) \equiv \mathcal{R}_{L}^{\mu \nu}(t)$. We can then rewrite Eq. (3.8) as

$$
\overline{\square_{\mathrm{R}}^{-1}\left[\tau^{\mu \nu}\right]}=\mathcal{D}^{-1}\left[\tau^{\mu \nu}\right]
$$

where the operator $\mathcal{D}^{-1}$ is naturally split into the two terms appearing in Eq. (3.8) as

$$
\mathcal{D}^{-1} \equiv \overline{\square_{\mathrm{R}}^{-1}}+\mathcal{H}
$$

We shall use this operator in the next Section devoted to the harmonicity conditions.

Note that the anti-symmetric multipolar waves such as $\mathcal{H}^{\mu \nu}$, given in Eq. (3.9), can be represented as a Fourier superposition of plane waves. We give here the relevant formulas without proof. The Fourier representation is arguably simpler and perhaps more physically appealing, but the alternative STF multipolar anti-symmetric formulation (3.9), which we mostly use in the present work, displays a structure that is often more useful in applications. Both forms are linked by

$$
\mathcal{H}^{\mu \nu}(\mathbf{x}, t)=\int d^{3} \mathbf{k}\left[A_{\text {out }}^{\mu \nu}(\mathbf{k}) e^{-2 \pi i k c t}+A_{\text {in }}^{\mu \nu}(\mathbf{k}) e^{2 \pi i k c t}\right] e^{2 \pi i \mathbf{k} \cdot \mathbf{x}},
$$

where the Fourier amplitudes $A_{\text {out }}^{\mu \nu}(\mathbf{k})$ and $A_{\text {in }}^{\mu \nu}(\mathbf{k})$ read, in terms of the multipolar functions $\mathcal{R}_{L}^{\mu \nu}(t)$, as

$$
\begin{aligned}
& A_{\text {out }}^{\mu \nu}(\mathbf{k})=-\frac{c}{2 k i} \sum_{\ell=0}^{+\infty} \frac{(-2 \pi i k)_{<L>}}{\ell !} F\left(\mathcal{R}_{L}^{\mu \nu}\right)(c k), \\
& A_{\mathrm{in}}^{\mu \nu}(\mathbf{k})=\frac{c}{2 k i} \sum_{\ell=0}^{+\infty} \frac{(-2 \pi i k)_{<L>}}{\ell !} F\left(\mathcal{R}_{L}^{\mu \nu}\right)(-c k),
\end{aligned}
$$


with $F\left(\mathcal{R}_{L}^{\mu \nu}\right)(\omega) \equiv \int_{-\infty}^{+\infty} d t e^{2 \pi i \omega t} \mathcal{R}_{L}^{\mu \nu}(t)$ representing the one-dimensional Fourier transform of $\mathcal{R}_{L}^{\mu \nu}(t)$. Here, we pose $k \equiv|\mathbf{k}|, k_{L} \equiv k_{i_{1}} \cdots k_{i_{\ell}}$ and the brackets surrounding indices denote the STF projection. Conversely, the function $\mathcal{R}_{L}^{\mu \nu}(t)$ is entirely determined by means of the Fourier amplitudes as ${ }^{6}$

$$
\mathcal{R}_{L}^{\mu \nu}(t)=(2 \ell+1) ! ! \int d^{3} \mathbf{k} \frac{(-2 \pi i k)_{<L>}}{(-2 \pi i k)^{2 \ell+1}}\left[-A_{\text {out }}^{\mu \nu}(\mathbf{k}) e^{-2 \pi i k c t}+A_{\text {in }}^{\mu \nu}(\mathbf{k}) e^{2 \pi i k c t}\right] .
$$

\section{HARMONICITY CONDITION}

In this Section, we prove that our post-Newtonian solution (3.7) is divergenceless as a consequence of the harmonicity condition, and thus constitutes an actual solution of the Einstein field equations. More precisely, we check that the conservation of the pseudotensor, Eq. (2.4), implies the satisfaction of the harmonicity conditions (2.7). This proof has already been done in paper II, but we present here the main steps of an alternative demonstration, using some of the tools employed there, and based directly on the new form (3.7) of the post-Newtonian expansion.

In order to verify the harmonicity condition, it is convenient to establish a sufficient property involving the operator $\mathcal{D}^{-1}$ introduced in Eq. (3.12). In fact, the solution written in the form (3.11) will be divergenceless as a consequence of $\partial_{\nu} \tau^{\mu \nu}=0$, if (mathematically speaking) we are allowed to commute the partial derivative $\partial_{\nu}$ with the $\mathcal{D}^{-1}$ operator, i.e. $\partial_{\nu} \mathcal{D}^{-1}\left[\tau^{\mu \nu}\right]=\mathcal{D}^{-1}\left[\partial_{\nu} \tau^{\mu \nu}\right]=0$. Let us define the following commutator: $\left[\partial_{\nu}, \mathcal{D}^{-1}\right] \equiv$ $\partial_{\nu} \mathcal{D}^{-1}-\mathcal{D}^{-1} \partial_{\nu}$. Thus, it is sufficient to prove that

$$
\left[\partial_{\nu}, \mathcal{D}^{-1}\right]=0
$$

The commutator corresponding to the partial time derivative is seen to be zero, $\left[\partial / \partial t, \mathcal{D}^{-1}\right]=0$, because the operations of taking the spatial integral and performing spatial differentiations which are involved in $\mathcal{D}^{-1}$ are obviously transparent to the time derivative. All what remains for us to prove is that $\left[\partial_{i}, \mathcal{D}^{-1}\right]=0$, for the partial space derivative

6 This relation is easily checked to be true by virtue of the formula

$$
\left(\frac{\partial}{c \partial t}\right)^{2 \ell+1} \mathcal{R}_{L}^{\mu \nu}(t)=(2 \ell+1) ! !(-1)^{\ell+1}\left(\hat{\partial}_{L} \mathcal{H}^{\mu \nu}\right)(\mathbf{0}, t)
$$

where $\mathcal{H}^{\mu \nu}(\mathbf{x}, t)$ is given by (3.9). 
$\partial_{i}=\partial / \partial x^{i}$. Therefore, we shall now show that

$$
\left[\partial_{i}, \mathcal{H}\right]=-\left[\partial_{i}, \overline{\square_{\mathrm{R}}^{-1}}\right]
$$

The first stage of our proof consists in evaluating $\partial_{i} \mathcal{D}^{-1}\left[\tau^{\mu \nu}\right]$ by decomposing the multiderivative acting on the anti-symmetric wave $\mathcal{H}_{L}^{\mu \nu} \equiv(2 r)^{-1}\left[\mathcal{R}_{L}^{\mu \nu}(t-r / c)-\mathcal{R}_{L}^{\mu \nu}(t+r / c)\right]$ into symmetric-trace-free (STF) pieces using the technical formula

$$
\partial_{i} \hat{\partial}_{L}=\hat{\partial}_{i L}+\frac{\ell}{2 \ell+1} \delta_{i\left\langle i_{\ell}\right.} \hat{\partial}_{L-1\rangle} \Delta
$$

where the brackets $\langle\cdots\rangle$ denote the STF operation for the indices they enclose. Each multipole order $\ell$ in the right-hand side of Eq. (3.9) is thus given by a sum of two terms. After changing appropriately the summation indices and bearing in mind that $\mathcal{H}_{L}^{\mu \nu}$ satisfies the homogeneous wave equation, so that $\Delta \mathcal{H}_{L}^{\mu \nu}=\left(c^{-1} \partial / \partial t\right)^{2} \mathcal{H}_{L}^{\mu \nu}$, we arrive at

$$
\begin{aligned}
\partial_{i} \mathcal{H}\left[\tau^{\mu \nu}\right] & =\sum_{\ell=0}^{+\infty} \frac{(-1)^{\ell}}{\ell !} \hat{\partial}_{L}\left\{\frac { 1 } { 2 r } \left[\ell \delta_{i\left\langle i_{\ell}\right.} \mathcal{R}_{L-1\rangle}\left[\tau^{\mu \nu}\right](t+r / c)\right.\right. \\
& \left.\left.+\frac{1}{2 \ell+3}\left(\frac{\partial}{c \partial t}\right)^{2} \mathcal{R}_{i L}\left[\tau^{\mu \nu}\right](t+r / c)-(\text { idem for the retarded wave })\right]\right\} .
\end{aligned}
$$

In a second stage, we shall work on the quantity $\mathcal{H}\left[\partial_{i} \tau^{\mu \nu}\right]$ in order to obtain an alternative expression involving $\mathcal{R}_{L}\left[\tau^{\mu \nu}\right]$ rather than $\mathcal{R}_{L}\left[\partial_{i} \tau^{\mu \nu}\right]$. The idea is to integrate by parts the integral on $\mathbf{x}^{\prime}$ defining $\mathcal{R}_{L}\left[\partial_{i} \tau^{\mu \nu}\right]$ as ${ }^{7}$

$$
\mathcal{R}_{L}\left[\partial_{i} \tau^{\mu \nu}\right]=\underset{B=0}{\mathrm{FP}} \int \frac{d^{3} \mathbf{x}^{\prime}}{-4 \pi}\left|\mathbf{x}^{\prime}\right|^{B} \hat{x}_{L}^{\prime} \int_{1}^{+\infty} d z \gamma_{\ell}(z) \mathcal{M}\left(\partial_{i} \tau_{\text {ns }}^{\mu \nu}\right)\left(\mathbf{x}^{\prime}, t-z\left|\mathbf{x}^{\prime}\right| / c\right),
$$

in a way that the source term does not contain any more derivatives. To begin with, we observe that $\mathcal{M}\left(\partial_{i} \tau_{\mathrm{ns}}^{\mu \nu}\right)$ is equal to a total space derivative, plus a term that turns out to involve a time derivative:

$\mathcal{M}\left(\partial_{i} \tau_{\mathrm{ns}}^{\mu \nu}\right)\left(\mathbf{x}^{\prime}, t-z\left|\mathbf{x}^{\prime}\right| / c\right)=\partial_{i}\left[\mathcal{M}\left(\tau_{\mathrm{ns}}^{\mu \nu}\right)\left(\mathbf{x}^{\prime}, t-z\left|\mathbf{x}^{\prime}\right| / c\right)\right]+z n_{i}^{\prime}\left(\frac{\partial}{c \partial t}\right) \mathcal{M}\left(\tau_{\mathrm{ns}}^{\mu \nu}\right)\left(\mathbf{x}^{\prime}, t-z\left|\mathbf{x}^{\prime}\right| / c\right)$.

The first contribution may be integrated by parts, which has the effect of transferring the operator $\partial_{i}$ onto the product $\left|\mathbf{x}^{\prime}\right|^{B} \hat{x}_{L}^{\prime}$. Its explicit action on the latter quantity reads

$$
\partial_{i}\left(\left|\mathbf{x}^{\prime}\right|^{B} \hat{x}_{L}^{\prime}\right)=\left[B n_{i}^{\prime} \hat{n}_{L}^{\prime}+(2 \ell+1)\left(n_{i}^{\prime} \hat{n}_{L}^{\prime}-\hat{n}_{i L}^{\prime}\right)\right]\left|\mathbf{x}^{\prime}\right|^{B+\ell-1} .
$$

\footnotetext{
${ }^{7}$ Henceforth we pose $r_{0}=1$.
} 
As $\tau_{\mathrm{ns}}^{\mu \nu}=0$ in the remote past, the surface term does not contribute. By applying the chain rule, we rewrite the second contribution in the right-hand side of Eq. (4.6) as $-\left(z x_{i}^{\prime} /\left|\mathbf{x}^{\prime}\right|^{2}\right)(\partial / \partial z) \mathcal{M}\left(\tau_{\mathrm{ns}}^{\mu \nu}\right)\left(\mathbf{x}^{\prime}, t-z\left|\mathbf{x}^{\prime}\right| / c\right)$. In particular, the contribution to the corresponding integral on $z$ may be integrated by parts in turn, and hence we are led to consider the first derivative $(d / d z)\left(z \gamma_{\ell}(z)\right)$, which verifies $(d / d z)\left(z \gamma_{\ell}(z)\right)=(2 \ell+1)\left(\gamma_{\ell}(z)-\gamma_{\ell-1}(z)\right)$. As a result, we get

$$
\begin{aligned}
& \mathcal{R}_{L}\left[\partial_{i} \tau^{\mu \nu}\right](t)=\mathrm{FP}_{B=0} \int \frac{d^{3} \mathbf{x}^{\prime}}{-4 \pi}\left|\mathbf{x}^{\prime}\right|^{B+\ell-1} \int_{1}^{+\infty} d z\left\{-B n_{i}^{\prime} \hat{n}_{L}^{\prime} \gamma_{\ell}(z)\right. \\
& \left.\quad+(2 \ell+1)\left[\left(\gamma_{\ell}(z)-\gamma_{\ell-1}(z)\right) \hat{n}_{i L}^{\prime}+\gamma_{\ell-1}(z)\left(\hat{n}_{i L}^{\prime}-n_{i}^{\prime} \hat{n}_{L}^{\prime}\right)\right]\right\} \mathcal{M}\left(\tau_{\mathrm{ns}}^{\mu \nu}\right)\left(\mathbf{x}^{\prime}, t-z\left|\mathbf{x}^{\prime}\right| / c\right) .
\end{aligned}
$$

The crucial point is that only a pole $\propto 1 / B$, generated by the radial integration, may give rise to a non-vanishing contribution for a source term proportional to $B$ in the limit $B \rightarrow 0$. Therefore, we may restrict the integration domain over the first term in the curly brackets above to a ball of radius $R$ centered at the origin without affecting its value. An immediate consequence is that $\mathcal{M}\left(\tau_{\text {ns }}^{\mu \nu}\right)$ can be replaced there by its post-Newtonian or near zone value $\overline{\mathcal{M}\left(\tau_{\text {ns }}^{\mu \nu}\right)}$. Moreover, we have $\overline{\mathcal{M}\left(\tau_{\text {ns }}^{\mu \nu}\right)}=\mathcal{M}\left(\bar{\tau}_{\text {ns }}^{\mu \nu}\right)$ by the matching between the inner and exterior fields (see paper II).

The second term entering the brackets of Eq. (4.8), in view of the relation $(d / d z)^{2}\left(\gamma_{\ell+1}(z)\right)=(2 \ell+1)(2 \ell+3)\left(\gamma_{\ell-1}(z)-\gamma_{\ell}(z)\right)$, is nothing but the second derivative of $\gamma_{\ell+1}(z)$ up to a constant factor. This derivative is handled by means of two successive integrations by parts, and the resulting derivatives that bear on $\mathcal{M}\left(\tau_{\mathrm{ns}}^{\mu \nu}\right)$ are transformed like

$$
\left(\frac{\partial}{\partial z}\right)^{2} \mathcal{M}\left(\tau_{\mathrm{ns}}^{\mu \nu}\right)\left(\mathbf{x}^{\prime}, t-z\left|\mathbf{x}^{\prime}\right| / c\right)=\left|\mathbf{x}^{\prime}\right|^{2}\left(\frac{\partial}{c \partial t}\right)^{2} \mathcal{M}\left(\tau_{\mathrm{ns}}^{\mu \nu}\right)\left(\mathbf{x}^{\prime}, t-z\left|\mathbf{x}^{\prime}\right| / c\right) .
$$

Taking also into account the fact that $\hat{n}_{i L}^{\prime}-n_{i}^{\prime} \hat{n}_{L}^{\prime}=-\ell \delta_{i\left\langle i_{\ell}\right.} \hat{n}_{L-1\rangle}^{\prime} /(2 \ell+1)$, this yields

$$
\begin{aligned}
\mathcal{R}_{L} & {\left[\partial_{i} \tau^{\mu \nu}\right]+\ell \delta_{i\left\langle i_{\ell}\right.} \mathcal{R}_{L-1\rangle}\left[\tau^{\mu \nu}\right]+\frac{1}{2 \ell+3}\left(\frac{d}{c d t}\right)^{2} \mathcal{R}_{i L}\left[\tau^{\mu \nu}\right] } \\
& =-\mathrm{FP}_{B=0} B \int \frac{d^{3} \mathbf{x}^{\prime}}{-4 \pi}\left|\mathbf{x}^{\prime}\right|^{B-2} x_{i}^{\prime} \hat{x}_{L}^{\prime} \int_{1}^{+\infty} d z \gamma_{\ell}(z) \mathcal{M}\left(\bar{\tau}_{\mathrm{ns}}^{\mu \nu}\right)\left(\mathbf{x}^{\prime}, t-z\left|\mathbf{x}^{\prime}\right| / c\right),
\end{aligned}
$$

which may be used to eliminate $\mathcal{R}_{L}\left[\partial_{i} \tau^{\mu \nu}\right]$ from the anti-symmetric wave $\mathcal{H}\left[\partial_{i} \tau^{\mu \nu}\right]$.

At this level, we note that at any finite post-Newtonian order, the structure of $\mathcal{M}\left(\bar{\tau}_{\text {ns }}^{\mu \nu}\right)$ is such (see paper II) that it yields a radial integral in the right-hand side of Eq. (4.10), that is decomposed into a sum of terms of the type $\int_{R}^{+\infty} d r^{\prime} r^{\prime B+q}$ with $q \in \mathbb{Z}$. Now, a 
useful lemma, already employed in Refs. 86, 9] and paper II, states that $\int_{0}^{+\infty} d r^{\prime} r^{\prime B+q}=0$ by analytic continuation in $B$. This implies that the right-hand side of Eq. (4.10) will give zero if we make the substitution $\tau_{\mathrm{ns}}^{\mu \nu} \rightarrow \tau_{\mathrm{s}}^{\mu \nu}$, which means that $\tau_{\mathrm{ns}}^{\mu \nu}$ can be replaced there by the complete pseudo tensor, $\tau^{\mu \nu}=\tau_{\mathrm{ns}}^{\mu \nu}+\tau_{\mathrm{s}}^{\mu \nu}$. Our desired commutator, i.e. the left-hand side of Eq. (4.2), follows immediately from Eqs. (3.9) and (4.10). We find

$$
\left[\partial_{i}, \mathcal{H}\right]\left[\tau^{\mu \nu}\right]=\sum_{\ell=0}^{+\infty} \frac{(-1)^{\ell}}{\ell !} \hat{\partial}_{L}\left\{\frac{\mathcal{S}_{L}^{i}\left[\tau^{\mu \nu}\right](t-r / c)-\mathcal{S}_{L}^{i}\left[\tau^{\mu \nu}\right](t+r / c)}{2 r}\right\},
$$

where

$$
\mathcal{S}_{L}^{i}\left[\tau^{\mu \nu}\right]=\underset{B=0}{\operatorname{FP}} B \int \frac{d^{3} \mathbf{x}^{\prime}}{-4 \pi}\left|\mathbf{x}^{\prime}\right|^{B-2} x_{i}^{\prime} \hat{x}_{L}^{\prime} \int_{1}^{+\infty} d z \gamma_{\ell}(z) \mathcal{M}\left(\bar{\tau}^{\mu \nu}\right)\left(\mathbf{x}^{\prime}, t-z\left|\mathbf{x}^{\prime}\right| / c\right) .
$$

In the last stage of our proof, we shall focus on the other commutator in the right-handside of Eq. (4.2), which is readily obtained by integration by parts as

$$
\left[\partial_{i}, \overline{\square_{\mathrm{R}}^{-1}}\right]\left[\tau^{\mu \nu}\right]=\sum_{k=0}^{+\infty} \frac{(-1)^{k}}{k !}\left(\frac{d}{c d t}\right)^{k} \underset{B=0}{\mathrm{FP}} B \int \frac{d^{3} \mathbf{x}^{\prime}}{-4 \pi}\left|\mathbf{x}^{\prime}\right|^{B-2} x^{i}\left|\mathbf{x}-\mathbf{x}^{\prime}\right|^{k-1} \bar{\tau}^{\mu \nu}\left(\mathbf{x}^{\prime}, t\right) .
$$

We shall put it under a form where it is immediate to see that it is the opposite of $\left[\partial_{i}, \mathcal{H}\right]$, which will complete our demonstration. Due to the presence of a $B$ factor, the only contributing terms are those that generate poles in the variable $B$ due to the divergent behavior of $\bar{\tau}^{\mu \nu}$ when $\left|\mathbf{x}^{\prime}\right| \rightarrow+\infty$. Thus, the integral depends only on the exterior domain, which can be chosen to lie outside a sphere of arbitrary radius $R$. If $R$ is large enough, we are allowed to replace $\left|\mathbf{x}-\mathbf{x}^{\prime}\right|^{k-1}$ and $\bar{\tau}^{\mu \nu}$ by their multipole expansions, so that

$$
\begin{aligned}
\underset{B=0}{\operatorname{FP}} B \int & \frac{d^{3} \mathbf{x}^{\prime}}{-4 \pi}\left|\mathbf{x}^{\prime}\right|^{B-2} x_{i}^{\prime}\left|\mathbf{x}-\mathbf{x}^{\prime}\right|^{k-1} \bar{\tau}^{\mu \nu}\left(\mathbf{x}^{\prime}, t\right)= \\
& \underset{B=0}{\operatorname{FP}} B \int_{\left|\mathbf{x}^{\prime}\right|>R} \frac{d^{3} \mathbf{x}^{\prime}}{-4 \pi}\left|\mathbf{x}^{\prime}\right|^{B-2} x_{i}^{\prime} \mathcal{M}\left(\left|\mathbf{x}-\mathbf{x}^{\prime}\right|^{k-1}\right) \mathcal{M}\left(\bar{\tau}^{\mu \nu}\right)\left(\mathbf{x}^{\prime}, t\right) .
\end{aligned}
$$

By virtue of the lemma mentioned above, we can change the integration over the domain $\left|\mathbf{x}^{\prime}\right|>R$ into an integration over the complementary domain $\left|\mathbf{x}^{\prime}\right|<R$ if we also change the sign. On the other hand, the expression of $\mathcal{M}\left(\left|\mathbf{x}-\mathbf{x}^{\prime}\right|^{s}\right)$ for $s \in \mathbb{N}$ is known explicitly. It is derived from the STF decomposition of the factors $x_{L}^{\prime}$ (see e.g. Eq. (2.2) in [24] or (A20a) in [7]) that enter the term of order $\ell$ in the series expansion of $\left|\mathbf{x}-\mathbf{x}^{\prime}\right|^{s}$ valid for $\left|\mathbf{x}^{\prime}\right|<|\mathbf{x}|$. We recover the same formula as Eq. (3.2) but for $k=s / 2$, namely

$$
\frac{\left|\mathbf{x}-\mathbf{x}^{\prime}\right|^{s}}{(s+1) !}=\sum_{\ell=0}^{+\infty} \frac{(-1)^{\ell}}{\ell !(2 \ell+1) ! !} \sum_{p=0}^{+\infty} \widetilde{\Delta^{-p}}\left(\hat{x}_{L}\right) \widetilde{\Delta^{-\frac{s}{2}+\ell+p}}\left(\hat{x}_{L}^{\prime}\right)
$$


provided that we define, for any integer $s$,

$$
\widetilde{\Delta^{-\frac{s}{2}}}\left(\hat{x}_{L}\right)=\frac{(2 \ell+1) ! !}{s ! !(2 \ell+s+1) ! !} r^{s} \hat{x}_{L}
$$

which generalizes the notation (2.18). We can transform (4.16), with the help of the Euler function $\mathrm{B}(p, q)=2 \int_{1}^{+\infty} d z z^{-2 p-2 q+1}\left(z^{2}-1\right)^{q-1}$, to

$$
\widetilde{\Delta^{-\frac{s}{2}}}\left(\hat{x}_{L}\right)=\frac{r^{s} \hat{x}_{L}}{s !} \frac{(-1)^{\ell+1}(2 \ell+1) ! !}{2^{\ell+1} \ell !} \mathrm{B}(-\ell-(s+1) / 2, \ell+1) .
$$

The appearance of the Euler function is of particular interest for our purpose, as it can simply be represented by an integral involving $\gamma_{\ell}(z)$. Indeed, we have $\mathrm{B}(-\ell-(s+1) / 2, \ell+1)=$ $2 \int_{1}^{+\infty} d z z^{s}\left(z^{2}-1\right)^{\ell}$, which immediately gives

$$
\widetilde{\Delta^{-\frac{s}{2}}}\left(\hat{x}_{L}\right)=\frac{r^{s} \hat{x}_{L}}{s !} \int_{1}^{+\infty} d z \gamma_{\ell}(z) z^{s} .
$$

Inserting the value of $\mathcal{M}\left(\left|\mathbf{x}-\mathbf{x}^{\prime}\right|^{k-1}\right)$ deduced from Eqs. (4.15)-(4.18) into the spatial integral with the radial variable $\left|\mathbf{x}^{\prime}\right|$ ranging from 0 to $R$ (taking into account the change of sign when going from the outer to the inner domain), we obtain

$$
\begin{aligned}
& {\left[\partial_{i}, \overline{\square_{\mathrm{R}}^{-1}}\right]\left[\tau^{\mu \nu}\right]=-\sum_{k, \ell, p \geq 0} \frac{(-1)^{k+\ell}}{(2 \ell+1) ! ! \ell !} \widetilde{\Delta^{-p}}\left(\hat{x}_{L}\right)} \\
& \times\left(\frac{d}{c d t}\right)^{k} \underset{B=0}{\mathrm{FP}} B \int_{\left|\mathbf{x}^{\prime}\right|<R} \frac{d^{3} \mathbf{x}^{\prime}}{-4 \pi} \frac{\left|\mathbf{x}^{\prime}\right|^{B+k-2 \ell-2 p-3} x_{i}^{\prime} \hat{x}_{L}^{\prime}}{(k-2 \ell-2 p-1) !} \int_{1}^{+\infty} d z \gamma_{\ell}(z) z^{k-2 \ell-2 p-1} \mathcal{M}\left(\bar{\tau}^{\mu \nu}\right)\left(\mathbf{x}^{\prime}, t\right) .
\end{aligned}
$$

Note that only the terms for which $k \geq 2 \ell+2 p+1$ contribute to the sum over $k$ since otherwise $1 /(k-2 \ell-2 p-1) !=0$. The divergences near $\left|\mathbf{x}^{\prime}\right|=0$ can be cured by means of the finite part regularization FP. We next permute the summation over $k$ and the integration on $z$. We easily recognize, from the summation over $k$, the Taylor expansion of $\mathcal{M}\left(\bar{\tau}^{\mu \nu}\right)\left(\mathbf{x}^{\prime}, t-z\left|\mathbf{x}^{\prime}\right| / c\right)$ when $c \rightarrow+\infty$. Finally, we obtain the relation

$$
\begin{aligned}
& {\left[\partial_{i}, \overline{\square_{\mathrm{R}}^{-1}}\right]\left[\tau^{\mu \nu}\right]=\sum_{\ell, p \geq 0} \frac{(-1)^{\ell}}{(2 \ell+1) ! ! \ell !} \widetilde{\Delta^{-p}}\left(\hat{x}_{L}\right)} \\
& \times\left(\frac{d}{c d t}\right)^{2 \ell+2 p+1} \operatorname{FP}_{B=0} B \int_{\left|\mathbf{x}^{\prime}\right| \leq R} \frac{d^{3} \mathbf{x}^{\prime}}{-4 \pi}\left|\mathbf{x}^{\prime}\right|^{B-2} x_{i}^{\prime} \hat{x}_{L}^{\prime} \int_{1}^{+\infty} d z \gamma_{\ell}(z) \mathcal{M}\left(\bar{\tau}^{\mu \nu}\right)\left(\mathbf{x}^{\prime}, t-z\left|\mathbf{x}^{\prime}\right| / c\right) .
\end{aligned}
$$

Thanks to the formula (3.1), which is valid for any function, we identify the right-hand side of (4.20) with the opposite of the commutator found in Eqs. (4.11)-(4.12), when it is developed as $c \rightarrow+\infty$. We can therefore conclude that

$$
\left[\partial_{i}, \mathcal{D}^{-1}\right] \equiv\left[\partial_{i}, \mathcal{H}\right]+\left[\partial_{i}, \overline{\square_{\mathrm{R}}^{-1}}\right]=0
$$


which, as we discussed above, achieves the proof that $\bar{h}^{\mu \nu}$ satisfies indeed the harmonicity conditions.

\section{CONCLUSION}

In conclusion, this work considers the general post-Newtonian approximation formalism for isolated, slow-moving matter systems. Our motivation is to justify the practical 3.5PN calculations of radiation reaction achieved in paper I (Ref. [1]). Our study follows from the previous investigation of paper II, in which a general scheme was proposed for the formal iteration of the post-Newtonian series in the source's near zone up to any post-Newtonian order. There, the expression comprised: (i) a particular solution to the flat wave equation, given in terms of a (well-defined) regularized solution, made with a finite part FP, to the iterated Poisson equation for a non-compact source, and (ii) a homogeneous solution, defined as a specific multipolar anti-symmetric wave, associated with radiation reaction effects. Here, we present an alternative, complementary expression for the gravitational field in the source's near-zone within the post-Newtonian approximation; notably, as in paper II, the expression incorporates fully the effects of the radiation reaction. It involves both particular and homogeneous solutions. In contrast to paper II, however, the particular solution in this instance represents a formal expansion of the retardations of the standard retarded integral, where each term has been suitably regularized using the FP procedure. The homogeneous second term, given in the form of a multipolar anti-symmetric wave, describes the non-linear contribution in the radiation reaction force of gravitational wave tails beginning at 4PN order $\sim 1 / c^{8}$. The "ordinary" radiation reaction effects are then contained in the odd-parity part of the retardations of the particular, inhomogeneous solution.

Consequently, as shown in Ref. [1], the radiation reaction effects in the local dynamics

of an isolated source up to and including the 3.5PN order $\sim 1 / c^{7}$ can be determined solely by considering the odd-parity part of the retardations in the standard retarded integral, following the appropriate regularization based on a FP prescription.

Finally and complementary to the proof given in paper II, we verify that our postNewtonian solution is divergenceless by demonstrating that the harmonic-coordinate condition is satisfied. Hence, the new expression is indeed a solution of the Einstein field equations. 
Together with useful technical relationships given throughout this paper, the new form for the metric in the near-zone enables significant insights into the character of the gravitational radiation reaction and contribution of tails for isolated matter systems in the post-Newtonian expansion of general relativity.

\section{Acknowledgments}

S.N. gratefully acknowledges the Entente Cordiale Scholarship and Robert Blair Fellowship (Corporation of London) for financial support during this work.

[1] S. Nissanke and L. Blanchet, Class. Quant. Grav. 22, 1007 (2005), gr-qc/0412018.

[2] B. Iyer and C. Will, Phys. Rev. Lett. 70, 113 (1993).

[3] P. Jaranowski and G. Schäfer, Phys. Rev. D 55, 4712 (1997).

[4] M. Pati and C. Will, Phys. Rev. D 65, 104008 (2002).

[5] C. Königsdörffer, G. Faye, and G. Schäfer, Phys. Rev. D 68, 044004 (2003).

[6] O. Poujade and L. Blanchet, Phys. Rev. D 65, 124020 (2002), gr-qc/0112057.

[7] L. Blanchet and T. Damour, Phil. Trans. Roy. Soc. Lond. A 320, 379 (1986).

[8] L. Blanchet, Phys. Rev. D 51, 2559 (1995), gr-qc/9501030.

[9] L. Blanchet, Class. Quant. Grav. 15, 1971 (1998), gr-qc/9801101.

[10] J. Plebanski and S. Bazanski, Acta Phys. Polonica 18, 307 (1959).

[11] S. Chandrasekhar and Y. Nutku, Astrophys. J. 158, 55 (1969).

[12] S. Chandrasekhar and F. Esposito, Astrophys. J. 160, 153 (1970).

[13] J. Anderson and T. DeCanio, Gen. Relativ. Gravit. 6, 197 (1975).

[14] J. Ehlers, Ann. N.Y. Acad. Sci. 336, 279 (1980).

[15] G. Kerlick, Gen. Relativ. Gravit. 12, 467 (1980).

[16] G. Kerlick, Gen. Relativ. Gravit. 12, 521 (1980).

[17] A. Papapetrou and B. Linet, Gen. Relativ. Gravit. 13, 335 (1981).

[18] V. Fock, Theory of space, time and gravitation (Pergamon, London, 1959).

[19] W. Burke, J. Math. Phys. 12, 401 (1971).

[20] L. Blanchet and T. Damour, Phys. Rev. D 37, 1410 (1988). 
[21] L. Blanchet and T. Damour, Annales Inst. H. Poincaré Phys. Théor. 50, 377 (1989).

[22] L. Blanchet, Phys. Rev. D 47, 4392 (1993).

[23] L. Blanchet, Phys. Rev. D 55, 714 (1997), gr-qc/9609049.

[24] K. Thorne, Rev. Mod. Phys. 52, 299 (1980). 